pressor cleavage ( $\lambda$ induction for example) or possibly both. In some cases (Weigle reactivation, filamentation) the mechanism is still obscure.

There is still, however, a niggling doubt about ascribing the proteolytic activity to the $\operatorname{rec} A^{+}$protein. As Roberts et al. state, they cannot exclude the possibility that a contaminating protease is present in their preparations even though they are chromatographically homogeneous. Consistent with this possibility is the observation that bacteria containing constitutive high levels of $\operatorname{rec} \mathrm{A}^{+}$protein due to the presence of the controlling mutation spr do not show constitutive $\lambda$ induction nor do crude extracts possess high ability to inactivate $\lambda$ repressor (Roberts et al. Proc. natn. Acad. Sci. U.S.A. 75, 4714; 1978). Several workers have suggested that the $\operatorname{rec} A^{+}$protein requires a cofactor to be active in prophage induction. It is not totally inconceivable that such a cofactor might be the protease and that it is present in greater amounts in tif mutants (although such speculation does present its own difficulties). Thus although the evidence that $\operatorname{rec} \mathrm{A}^{+}$protein is both a protease and a DNA annealase may be sufficient to sustain the idea as a working hypothesis, more definitive evidence must be sought and the possibility of further revelations of the functions of this remarkable protein cannot be excluded.

\section{Chamber crystals in the stratosphere}

\section{from Brian A. Thrush}

ONE aspect of stratospheric chemistry which has been largely overlooked in the recent flurry of activity has been the role of the aerosol particles. These form the Junge layer in the lower stratosphere and have their peak density at an altitude of about $18 \mathrm{~km}$. Although these particles, which have sizes in the range $0.05-1 \mu \mathrm{m}$, number only about one per $\mathrm{cm}^{3}$, the amount of material they contain is comparable with the amounts of important trace materials in the lower stratosphere, such as the nitrogen oxides. If these species reacted efficiently with the aerosol particles, this process could be significant to the chemistry of the stratosphere. Chemical analysis shows this aerosol to contain ammonium sulphate, accompanied by persulphate and perhaps chloride, but recently Farlow et al. (J. geophys. Res. 83, 620711 ; 1978) have tentatively identified two nitrosyl sulphuric acids, $\mathrm{NOHSO}_{4}$ and $\mathrm{NOHS}_{2} \mathrm{O}_{7}$ by electron diffraction studies of aerosol particles collected by U-2 aircraft.

\title{
Poles apart?
}

\section{from Clive Lloyd}

THERE is still much to be learned about the mitotic apparatus, especially the nature of spindle organisers. Such microtubule-organising centres (MTOCs) are believed to exist at each spindle pole, where, by nucleating the assembly of microtubules, they help construct half-spindles along which sister chromatids are guided to opposite poles. In vitro studies confirm that microtubules can self-assemble, but the value of MTOCs is that they localise assembly and so help control planes of division important for shaping tissues. The most familiar MTOC is associated with the centriole which is a highly structured organelle resembling a vestigial flagellum. It is noteworthy, though, that amorphous pericentriolar material would seem to be the spindle organiser in in vitro polymerisation studies on Chinese hamster ovary cells so, if nothing else, the centriole provides a convenient signpost for such associated material (Gould \& Borisy J. Cell Biol. 73, 601; 1977).

Definite pole bodies are to be seen in algae and fungi, but in higher plants we are confronted with the problem that such structures are not visualised in ultrastructural studies of dividing cells. This has forced the alternative view that accumulated endoplasmic reticulum seen at the poles could, by sequestering calcium, precipitate localised formation of microtubules (Hepler in Mechanisms \& Control of Cell Division (eds Rost \& Gifford) 1977), although it is admittedly difficult to see how, in the absence of a template, the number, pattern and precise location of microtubules could be controlled.

However, a recent study on the effect of a herbicide on 3T3 fibroblasts rekindles interest in presumptive spindle organisers since the drug is well known to produce mitotic abnormalities in plants. Oliver et al.

The stratosphere is very dry, containing only a few parts per million of water, and it is interesting to note that $\mathrm{NOHSO}_{4}$ is very stable under such conditions. It is familiar to chemists as forming the 'chamber crystals' which appear in the lead chamber process for making sulphuric acid. They are formed from sulphur dioxide and nitrogen dioxide $\left(\mathrm{NO}_{2}\right)$ when insufficient water is present. The authors point out that if this compound comprised $20 \%$ of the sulphate in stratospheric aerosol then this would contain about one third of the amount of nitric oxide (NO) which is present at these altitudes.
(Expl Cell Res. 116, 229; 1978), showed that chloro-isopropyl N-phenyl carbamate (CIPC) causes the formation of multinocleate cells by inducing multipolar mitotic spindles. In vitro polymerisation studies indicated that CIPC, unlike colchicine, did not interfere with tubulin assembly and so it was concluded that the herbicide had affected spindle MTOCs rather than microtubules per se. This report on animal cells echoes the earlier study of Hepler and Jackson (J. Cell Sci. 5, 727; 1969) on dividing nuclei in the endosperm of the African blood lilly. Whereas colchicine caused microtubule disassembly, IPC did not, but with the latter treatment multipolar spindles were formed. However, although microtubules were seen to radiate from some central locus, electron microscopy failed to give shape to the spindle pole. Following this, Coss \& Pickett-Heaps ( $J$. Cell Biol. 63, 84; 1974) used IPC to examine division in the green alga, Oedogonium cardiacum. They, too, found that multinucleate cells were formed, suggesting that the herbicide increased the number of MTOCs. No such bodies could be identified in thin sections but with high voltage electron microscopy on thick sections the picture was quite different, for morphologically distinctive spindle pole bodies were revealed in both normal and drug-treated mitoses. This family of reactions on representatives of animal cells, higher plants and algae indicates that some divisible MTOC is being affected by the herbicides but it remains to be determined whether endoplasmic reticulum, as opposed to some other associated, perhaps amorphous, nucleation site, is being perturbed in higher plant cells.

Clive Lloyd is in the Biosciences Division, Unilever Research, Bedford.

Because the nitrogen oxides NO and $\mathrm{NO}$, catalytically destroy ozone in the stratosphere, any process which affects their concentrations is potentially important. In fact, neither the measured ratio of $\mathrm{NO}$ and $\mathrm{NO}_{2}$ to nitric acid ( $\mathrm{HONO}_{2}$ ) nor the failure to detect pernitric acid $\left(\mathrm{HO}_{2} \mathrm{NO}_{2}\right)$ in the lower stratosphere agree well with predictions based on known homogeneous chemical reactions. Reactions involving aerosol particles may be implicated here.

In the stratosphere, these nitrogen compounds come mainly from nitrous oxide $\left(\mathrm{N}_{2} \mathrm{O}\right)$ derived from denitrification processes and could therefore be 